EGU21-3523, updated on 30 Mar 2021

https://doi.org/10.5194/egusphere-egu21-3523

EGU General Assembly 2021

(c) Author(s) 2021. This work is distributed under

the Creative Commons Attribution 4.0 License.

\title{
ETAS Space time modelling of Chile induced seismicity using covariates.
}

\author{
Marcello Chiodii, ${ }^{1,2}$, Orietta Nicolis ${ }^{3}$, Giada Adelfio ${ }^{1,2}$, Nicoletta D'angelo ${ }^{1}$, and Alex Gonzàlez ${ }^{3}$ \\ ${ }^{1}$ Department of Economics, Business and Statistics, University of Palermo, Italy (marcello.chiodi@unipa.it) \\ ${ }^{2}$ INGV (National Institute of Geophysics and Volcanology) Italy \\ ${ }^{3}$ Universidad Andres Bello Chile
}

Chilean seismic activity is among the strongest ones in the world. As already shown in previous papers, seismic activity can be usefully described by a space-time branching process, like the ETAS (Epidemic Type Aftershock Sequences) model, which is a semiparametric model with a large time scale component for the background seismicity and a small time scale component for the induced seismicity. The large-scale component intensity function is usually estimated by nonparametric techniques, specifically in our paper we used the Forward Likelihood Predictive approach (FLP); the induced seismicity is modelled with a parametric space-time function. In classical ETAS models the expected number of induced events depends only on the magnitude of the main event. From a statistical point of view, forecast of induced seismicity can be performed in the days following a big event; of course the estimation of this component is very important to forecast the evolution, in space and time domain, of a seismic sequence. Together with magnitude, to explain the expected number of induced events we also used other covariates. According to this formulation, the expected number of events induced by event $E_{i}$ is a function of a linear predictor $\eta_{i}=\mathbf{x}_{i} \boldsymbol{\beta}$, where $\mathbf{x}_{i}$ is the vector of covariates observed for the $i$-th event (the first is usually the magnitude $m_{i}$ ), and $\boldsymbol{\beta}$ is a vector of parameters to be estimated together with the other parametric and nonparametric components of the ETAS model. We obtained some interesting result using some covariates related to the depth of events and to some GPS measurement, corresponding to earth movement observed before main events. We find that some of these models can improve the description and the forecasting of the induced seismicity in Chile, after a subdivision of the country in different spatial regions. We used open-source software (R package etasFLP) to perform the semiparametric estimation of the ETAS model with covariates. 